\title{
Gaia: An astrometric solution Jonah Hansen
}

\section{Abstract}

The European Space Agency (ESA) launched a mission, named Gaia, that recently released its first catalogue of data. In this essay, I detail why Gaia is important and how it is revolutionary to the field of astrometry (the study of the position and motions of celestial objects). I also describe various techniques for finding exoplanets and how no technique, with the exception of astrometry, can find the true mass of an exoplanet. Finally, I explain how Gaia may be one of the first missions to detect exoplanets with the astrometric technique and, by extension, find the true mass and distribution of exoplanets.

\section{Introduction}

Since the early days of science, the study of the positions of celestial objects, known as astrometry, has been a vital component of many advances and discoveries in astronomy. However, most instruments used to measure a stellar object's position have not had the precision required to discover and understand crucial theories concerning many astronomical bodies. The European Space Agency's recent space mission, Gaia, aims to revolutionise the field by providing an accurate and precise $3 \mathrm{D}$ map of all stars up to 
a G magnitude of 20.7 mag. ${ }^{1}$ With this extensive survey, it is likely that many discoveries will be made; in particular, the very first detection of exoplanets via the astrometric technique.

\section{The Gaia mission}

A star's position in the sky can be reported with six standard kinematic parameters: angular position $(\alpha, \delta)$, proper motion $\left(\mu_{\alpha^{\prime}}, \mu_{\delta}\right)$, parallax $(\pi)$, and radial velocity $\left(R_{v}\right)$. With many new, innovative techniques and advances in astronomical instrumentation, most of these parameters have been determined accurately with acceptable uncertainties. However, until 1997, finding the accurate parallax of a celestial object from the ground was extremely challenging, due to the major disturbances caused by Earth's atmosphere. Systematic errors also arose due to the need to correct from the relative ground-based parallax to absolute parallax. ${ }^{2}$

In 1997 the European Space Agency launched a satellite named HIPPARCOS, a reference to the father of trigonometry, which was designed to measure the absolute parallax of 117,995 objects with uncertainties of around a milliarcsecond. ${ }^{3}$ These new accurate parallaxes proved invaluable to the field of astrometry and astronomy as a whole, allowing insights into the structure and formation of the Milky Way Galaxy. Nevertheless, HIPPARCOS had a very limited sample size, and so a new mission was devised to increase the number of stars with accurately known positions from 100,000 to over 1 billion. ${ }^{4}$

This new mission, called Gaia, was launched in 2013 with the aim to derive highly accurate kinematic parameters for all stars up to a magnitude of $G \approx$ 20.7 mag. ${ }^{5}$ Gaia's planned precision will completely change the field, providing uncertainties from $10 \mu$ as for $G \approx 13 \mathrm{mag}$ to $600 \mu$ as at $G \approx 20 \mathrm{mag}$ for all

1 Gaia Collaboration, Tino Prusti et al. 2016. 'The Gaia mission.' ArXiv e-prints, September. doi:10.1051/0004-6361/201629272.

2 Ibid.

3 Ibid.

4 Gaia Collaboration, Anthony G.A. Brown et al. 2016. 'Gaia Data Release 1. Summary of the astrometric, photometric and survey properties.' ArXiv e-prints, September. doi: 10.1051/00046361/201629512.

5 Gaia Collaboration, 'The Gaia mission'. 
kinematic parameters excluding radial velocity. ${ }^{6}$ In addition, it will perform photometric and spectroscopic surveys of all sources, providing one of the biggest and most in-depth surveys of the Milky Way galaxy so far.

The Gaia spacecraft is located at the second Lagrange point (L2) of the Sun-Earth-Moon system, which has multiple benefits for the lifetime of the spacecraft, including stable temperatures and minimal radiation interference. ${ }^{7}$ At the L2 point, Gaia will have maximum viewing efficiency, with the Earth, Moon and Sun located outside of the field of view. In order for it to scan the sky in a highly efficient manner that allows uniform coverage, the spacecraft was put into a Lissajous-type orbit, with an orbital period of around 180 days. ${ }^{8}$

There are many astronomical fields in which Gaia's astrometric survey will be of vital importance. One such field is galactic structure and dynamics. Through analysing the distribution and kinematics of both matter and dark matter in the Milky Way, Gaia will help to shed light on how the galaxy was formed and how it evolved into what is observed today. ${ }^{9}$ The fundamental question 'What is dark matter?' will also be addressed to some extent, potentially providing ground-breaking discoveries relevant to not just astronomy, but to physics as a whole. Other scientific mission objectives can be found in the Gaia Collaboration's 'The Gaia Mission' paper. ${ }^{10}$

However, what is one of the newest and most exciting prospects is in the field of exoplanets, where Gaia will assist in locating the first astrometrically determined exoplanets.

\section{Exoplanet detection}

Currently, there are multiple ways of detecting exoplanets, each with their own strengths and weaknesses. One of the first, and most used until recently, is the radial velocity technique, in which a star's radial velocity is measured using its spectrum's Doppler shift. If a planet were to orbit a star, then both

$\begin{array}{ll}6 & \text { Ibid. } \\ 7 & \text { Ibid. } \\ 8 & \text { Ibid. } \\ 9 & \text { Ibid. } \\ 10 & \text { Ibid. }\end{array}$ 
that star and planet would orbit their barycentre, causing the star to move along its radial velocity axis slightly. The radial velocity semi-amplitude, $K$, is given by the following equation derived by Lovis and Fischer:

Equation $1^{11}$

$K=\sqrt{\frac{G}{1-e^{2}}} \frac{m_{p} \sin i}{\left(m_{s}+m_{p}\right)^{\frac{2}{3}}}$

Where $m_{3} m_{p}$ is the mass of the star and planet respectively, $P$ is the period, $i$ is the inclination, and $e$ is the eccentricity of the orbit. As seen above, the true mass of the planet cannot be found due to the unknown inclination angle of the orbit, inhibiting studies on individual exoplanet systems. Another downfall is that the amplitude is inversely related to the period, and hence has a bias for systems with small periods, that is planets close to their host star.

In the past few years, NASA's Kepler spacecraft has detected at least 1,284 exoplanets using the transit technique, where a planet blocks the light of the host star as it passes in front of it. ${ }^{12}$ While the transit technique by itself cannot determine the mass of a planet, it ensures that if the detection is followed up by a radial velocity investigation, the inclination of the system's orbit will be equal to $1 .{ }^{13}$ However, as seen in Equation 1, the planet's mass is still tied to the mass of the whole system, rendering it near impossible to determine the true mass without knowing external information about the star itself. Furthermore, the transit technique only produces results for planets with short periods and with an inclination of 1 . This reduces the number of exoplanets with discoverable masses considerably.

This lack of a technique that reliably determines a planet's true mass is hindering the growth of the exoplanet field, preventing studies into the individual properties of exoplanets. The astrometric technique solves this problem by introducing a method that removes the dependence of inclination on an exoplanet system's mass. Similar to radial velocity, the astrometric method infers an exoplanet from the observations of a parent star orbiting

11 Ibid.

12 Timothy D. Morton et al. 2016. 'False Positive Probabilities for all Kepler Obects of Interest.' The Astrophysical Journal 882(2). doi:10.3847/0004-637X/822/2/86.

13 Wesley A. Traub and Ben R. Oppenheimer. 2010. 'Direct Imaging of Exoplanets.' In Exoplanets, ed. Sara Seager. Tucson: University of Arizona Press. 
the barycentre. ${ }^{14}$ However, while the radial velocity technique only observes the radial component of the stellar motion, astrometry measures the projected orbit as an ellipse in the plane of the sky.

As provided by Perryman et al., the astrometric signature, which is the signal given in angular measure, is calculated from the formula below:

\section{Equation $2^{15}$}

$\alpha=\left(\frac{M_{p}}{M_{s}}\right)\left(\frac{a_{p}}{1 A U}\right)\left(\frac{d}{1 p c}\right)^{-1} \operatorname{arcsec}$

where $a_{p}$ is the semi-major axis of the orbit ellipse with respect to the barycentre. As shown in Equation 2, the mass of the planet can be found without respect to its inclination. The full Keplerian orbit can also be determined from an astrometric measurement, as long as the object is viewed with respect to an absolute reference frame, as is the case with Gaia. ${ }^{16}$

\section{The advantages of astrometry}

The astrometric technique removes the bias towards planets with small periods; in fact, the signature scales linearly with a planet's distance from the parent star. This provides a unique opportunity to discover planets beyond the limit of radial velocity, in particular around the snow line.

The study of planets around the snow line, such as Jupiter in our own solar system, is crucial to the study of solar system formation, as the current formation model of the solar system states that gas giants can only form beyond this line. ${ }^{17}$ The validity of this model was threatened by the discovery of hot Jupiters - planets that are very close to their parent star but with very large radii. The current explanation for these planets is that they migrated

14 Andreas Quirrenbach. 2010. 'Astrometric Dectection and Characterization of Exoplanets.' in Exoplanets, ed. Sara Seager. Tucson: University of Arizona Press.

15 Gaia Collaboration. 2016. 'Gaia Data Release 1. Summary of the astrometric, photometric and survey properties'.

16 Michael Perryman et al. 2014. 'Astrometric Exoplanet Detection with Gaia.' The Astrophysics Journal 797(1). dx.doi.org/10.1088/0004-637X/797/1/14.

17 EDx Online Course Notes, 2015. 'Combined Reference Notes: Exoplanets Course.' courses. edx.org/courses/course-v1:ANUx+ANU-ASTRO2x+2T2016/info. 
inward due to ripples in the proto-planetary disk but, if that is the case, either most giant planets are hot Jupiters, or the theory is incomplete. ${ }^{18}$ Studying the planets beyond this line is crucial to understanding these formation theories, and astrometry is currently the only technique posed to answer these questions.

However, perhaps the greatest use of astrometry is in complementing the other exoplanet-finding techniques, such as direct imaging or radial velocity. Once a star with an exoplanet is found via one of these techniques, a follow up with astrometry would provide vital data about the planet, including its true mass and its three-dimensional orbit. ${ }^{19}$ Once the true mass has been found, the density, surface gravity and atmosphere can be determined, with spectral investigations playing a role in the characterisation of the latter. This presents a very exciting opportunity to examine candidates for Earth-like planets in the habitable zones of stars to a greater extent than is possible today, possibly resulting in the discovery of the first exoplanet that could support life. As also revealed by Morton et al., the Kepler mission has reported many potential false-positives. These false-positives can be followed up through astrometric techniques, validating that the object is an exoplanet and not an eclipsing binary. ${ }^{20}$

Astrometry is not without its weaknesses, primary among them being the sensitivity requirement of the instrument. To detect large planets, the astrometric signature required is $.1 \mathrm{mas}$, and for Earth-sized planets the astrometric signature can be in the sub $1 \mu$ as range. ${ }^{21}$ In addition, for planets with large periods, which astrometry has a bias for, the observing time must become increasingly long to characterise the exoplanets to the same precision as planets with smaller periods. While Gaia will certainly provide astrometric sensitivity to detect massive planets with large semi-major axes, such as Jupiter and Saturn, a sizable proportion of Earth-sized planets may be out of range for the mission. ${ }^{22}$ Nevertheless, Gaia will be a vital component for the near future of exoplanet research.

18 Ibid.

19 Michael Shao et al. 2009. 'Astrometric Detection of Earthlike Planets.' Astro2010: The Astronomy and Astrophysics Decadal Survey, Science White Papers, 271, arxiv.org/abs/0904.0965. 20 Ibid.

21 Andreas Quirrenbach. 2010. 'Astrometric Dectection and Characterization of Exoplanets.' in Exoplanets, ed. Sara Seager. Tucson: University of Arizona Press.

22 Gaia Collaboration, 'The Gaia mission'. 
One of the strengths of Gaia's mission is that as well as determining the distance and discerning the orbital motions for currently known exoplanet systems, it will take a survey of stars with varying types and ages. ${ }^{23}$ This will produce an unbiased exoplanet search over a range of orbital periods that have not been well studied.

The precision to which the stellar parameters are measured is also critical, and hence, due to Gaia's unprecedented astrometric sensitivity, it will be the first satellite to be precise enough to identify exoplanets using astrometric identification. Perryman et al. calculate that based on Gaia's astrometric capabilities, around 21,000 planets at a distance up to $500 \mathrm{pc}$ will be detected, of which 1,000-1,500 will be detectable around M-dwarfs at a distance around $100 \mathrm{pc}$. In addition, most of these will have clearly defined orbits, and while some of the long period systems may be poorly constrained, these can be improved with follow-up radial velocity observations.

With the large, unbiased survey of stars, Gaia is expected to provide a more reliable exoplanet population distribution, detailing the commonality of hot Jupiters and super-Earths, as well as detecting the frequency of exoplanets based on semi-major axis and host star type. ${ }^{24}$ Furthermore, as detailed previously, Gaia should provide important revelations into the process of gas giant formation, possibly solving the problem concerning the large amount of hot Jupiters and testing the migration theory.

Perryman et al. also make a suggestion for the number of planets detected if Gaia's mission length were to extend beyond the initial five years to a 10-year mission. Were this to be the case, the number of exoplanets detected and the quality of the determined orbits would drastically improve, producing an estimated 70,000 exoplanets over the mission period.

\section{Conclusion}

ESA's Gaia mission is sure to leave its mark on the field of astrometry and astronomy in general. Through its unprecedented precision of measuring stellar positions and velocities, it will produce vital data that will help in our

23 Michael Perryman et al. 2014. 'Astrometric Exoplanet Detection with Gaia.' The Astrophysical Journal 797(1). dx.doi.org/10.1088/0004-637X/797/1/14.

24 Ibid. 
understanding of the galaxy, specifically in the field of astrometric exoplanet detection. The discovery of around 21,000 exoplanets of stars of all types and sizes will allow astronomers to glean information about exoplanet formation, know the distribution of exoplanets, and it may even result in the holy grail of exoplanet astronomy: the detection of a planet that could sustain life.

\section{References}

Edx Online Course Notes. 2015. 'Combined Reference Notes: Exoplanets Course.' courses.edx.org/courses/course-v1:ANUx+ANUASTRO2x+2T2016/info.

Gaia Collaboration, Brown, Anthony G.A., Antonella Vallenari, Tino Prusti, Jos de Bruijne, François Mignard, Ronald Drimmel et al. 2016. 'Gaia Data Release 1. Summary of the astrometric, photometric and survey properties.' ArXiv e-prints, September. doi:10.1051/0004-6361/201629512.

Gaia Collaboration, Prusti, Tino, Anthony G.A. Brown, Jos H. J de Bruijne, Antonella Vallenari, Carine Babusiaux, Coryn A. L. Bailer-Jones, Ulrich Bastian et al. 2016. 'The Gaia mission.' ArXiv e-prints, September. doi:10.1051/0004-6361/201629272.

Lovis, Christophe and Debra A. Fischer. 2010. 'Radial Velocity Techniques for Exoplanets.' In Exoplanets, edited by Sara Seager. Tucson: University of Arizona Press, 2010. doi.org/10.1088/0004-637X/797/1/14.

Morton, Timothy D., Stephen T. Bryson, Jeffery L. Coughlin, Jason F. Rowe, Ganesh Ravichandran, Erik A. Petigura, Michael R. Haas and Natalie M. Batalha. 2016. 'False Positive Probabilities for all Kepler Objects of Interest.' The Astrophysical Journal 822(2). doi:10.3847/0004-637X/822/2/86.

Perryman, Michael, Joel Hartman, Gáspár Á. Bakos, and Lennart Lindegren. 'Astrometric Exoplanet Detection with Gaia.' The Astrophysical Journal 797(1). dx.doi.org/10.1088/0004-637X/797/1/14.

Quirrenbach, Andreas. 2010. 'Astrometric Detection and Characterization of Exoplanets.' In Exoplanets, edited by Sara Seager. Tuscon: University of Arizona Press. 
Shao, Michael, Joseph H. Catanzarite, Stephen J. Edberg, Alain Leger, Fabien Malbet, Didier Queloz, Matthew W. Muterspaugh et al. 2009. 'Astrometric Detection of Earthlike Planets.' Astro2010: The Astronomy and Astrophysics Decadal Survey, Science White Papers, 271. arxiv.org/ abs/0904.0965.

Traub, Wesley A. and Ben R. Oppenheimer. 2010. 'Direct Imaging of Exoplanets.' In Exoplanets, edited by Sara Seager. Tuscon: University of Arizona Press. 
This text is taken from Merici, Volume 2, 2016, edited by Matthew Rogers, published 2017 by ANU eView, The Australian National University,

Canberra, Australia. 